\title{
High Reference Range in Numeric Format
}

National Cancer Institute

\section{Source}

National Cancer Institute. High Reference Range in Numeric Format. NCI Thesaurus.

Code C121937.

The upper limit of the test reference range, reported in numerals. 